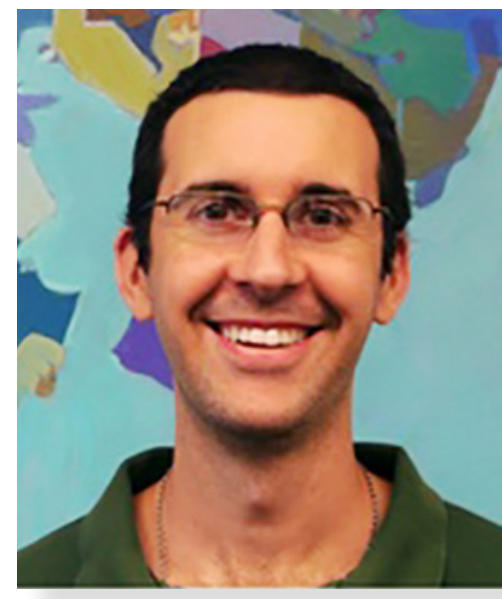

Guillermo Marini*

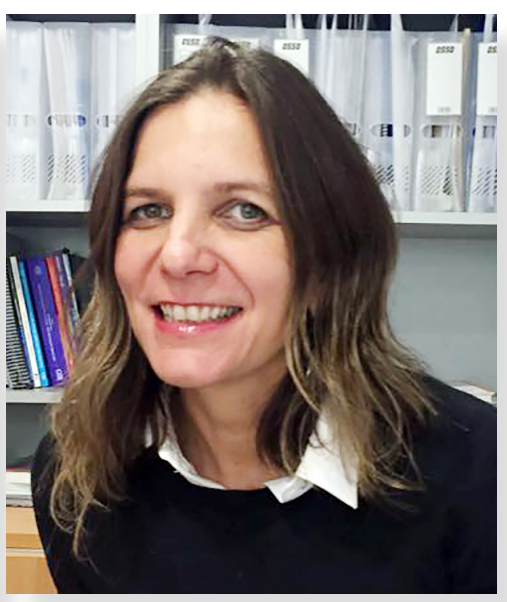

Magdalena Müller Araya**

\section{Ensayo de Posición Pedagógica: Una estrategia para visibilizar la formación docente}

\section{Pedagogical Position Essay: A strategy to make teacher education visible}

Recibido: 19-10-18 Aprobado: 20-12-18

\title{
Resumen
}

El Ensayo de Posición Pedagógica (EPP) constituye una estrategia de formación, en la cual el profesor redacta los principios que guían su labor, y los reescribe según se suceden experiencias clave. Este artículo presenta el diseño, implementación y evaluación del EPP con estudiantes del Programa de Formación Pedagógica de la Pontificia Universidad Católica de Chile. Su objetivo general consiste en evaluar la progresión del EPP a lo largo de la trayectoria de dichos docentes en formación. Mediante un estudio comparativo y de entrevistas semiestructuradas se analizan tres instancias del EPP: 1. cuando los estudiantes carecen de experiencia docente, 2. al iniciar sus prácticas docentes, 3. hacia el final del Programa de Formación Pedagógica. El EPP contribuye a revelar el circuito complejo entre experiencia de vida, cursos académicos, y contexto laboral mientras los profesores se adentran en su profesión.

\section{Abstract}

The Pedagogical Position Essay (EPP) constitutes an educational strategy, in which teachers write down the basic principles that guide their work, and rewrite them according to key experiences. This article presents the design, implementation and evaluation of the EPP with students of the High School Teacher Training Program at Pontificia Universidad Católica de Chile. Its general objective is to evaluate the progression of the EPP along the educational trajectory of these teachers. Through a comparative study and semi-structured interviews, three instances of the EPP are analyzed: 1. when students have no teaching experience, 2. at the beginning of their practicum work, 3. towards the end of their Teacher Training Program. The EPP contributes to revealing the complex circuit between life experience, academic courses and work context of teachers as they navigate their profession.

\section{Palabras clave}

ensayo; experiencia pedagógica; experiencia profesional; formación inicial de profesores; formación profesional

\section{Keywords}

essay; pedagogical experience; professional experience; initial teacher training; vocational training

\footnotetext{
*Guillermo Marini: PhD in Philosophy and Education por Columbia University, y EdM in Arts in Education por Harvard University, cursó su pregrado en Filosofía en la Pontificia Universidad Católica Argentina. Actualmente, se desempeña como profesor asociado en el Departamento de Teoría y Política de la Facultad de Educación UC. Para contactar al autor: gmarini@uc.cl. https://uc-cl.academia.edu/GuillermoMarini

**Magdalena Müller Araya: Doctora en Psicología por la Pontificia Universidad Católica de Chile, y Licenciada en Educación Diferencial con mención en Trastornos Específicos del Aprendizaje de la Pontificia Universidad Católica de Valparaíso. Actualmente está cargo del proyecto: Red Educacional UC que tiene como objetivo fortalecer el vínculo entre la Universidad y los colegios en que los profesores en formación realizan sus prácticas profesionales (2009 a la fecha). Para contactar a la autora: mbmuller@uc.cl
} 


\section{Introducción}

El ejercicio de reflexión que surge a partir de la elaboración del EPP es una oportunidad de aprendizaje en la trayectoria formativa para que los estudiantes se vinculen con sus ideas y creencias sobre la educación, y la transformación de las mismas a lo largo de su propia carrera profesional. En este sentido, incluir la redacción y reescritura del EPP como una actividad formativa dentro de instancias regulares del currículum, favoreciendo además que los estudiantes compartan sus escritos y reflexionen acerca de los cambios que van observando, es un aporte que permite a los docentes mantenerse vinculados al sentido de su profesión.

Según Donald Schön (1983), los profesionales exhiben un tipo de reflexión que los distingue de las prácticas de un novel en dos aspectos fundamentales: tienen mayores recursos para valorar el potencial de lo inesperado, es decir, están capacitados para apreciar las posibilidades de los imprevistos en su trabajo; y son capaces de reformular sus propias presunciones y decidir cómo llevar adelante su trabajo de modos nuevos. Amodo de ejemplo, Schön presenta al "profesor reflexivo" como aquel profesional de la educación cuya reflexión está "directamente comprometida con resolver los problemas que los profesionales deben enfrentar en las pantanosas arenas de la práctica" (Zeichner, 1996, p. 221).

Si bien la noción de "profesor reflexivo" ha sido asumida por gran parte del campo docente como expresión elocuente para caracterizar las exigencias de la cotidianidad pedagógica, existen reparos sobre la viabilidad de enseñar tal reflexión. Por un lado, pretender reflexionar no garantiza hacerlo bien y de modo coherente (Ward y McClotter, 2004). Por otra parte, quienes están a cargo de la formación de profesores deberían realizar primero un cambio paradigmático respecto de qué significa enseñar a ser un profesor reflexivo antes de esperar que los futuros profesores sean capaces de mostrar por sí solos cómo se logra tal reflexión.

A partir de estas críticas, dos discípulos de Schön, Kenneth Zeichner y Thomas Russell, proponen que las facultades de educación y los institutos de formación docentes son los mayores responsables de modelar las habilidades de reflexión de los futuros profesores (Zeichner, 2010; Kretchmar y Zeichner, 2016; Russell y Munby, 1991). Este modelamiento se daría específicamente en la articulación entre cursos académicos y prácticas profesionales, forjando un lugar desde donde el futuro profesor puede aprender a explicitar y reformular los principios que guiarán su trabajo, a partir de criterios claros (Thomas, 2012; Zeichner, 2012). En otras palabras, el enlace entre cursos y prácticas sería el espacio propicio para intencionar y desarrollar las capacidades de reflexión de los futuros profesores, y relevar la transformación de estudiante de pedagogía a profesor (Montell, 2003b; Müller, Álamos, Meckes, Sanyal y Cox, 2016; Staal, Klette y Hamerness, 2018).

El aporte de Russell (2005a) es particularmente relevante para este artículo ya que propone que los futuros profesores redacten un breve texto que puedan revisar a lo largo de su formación, como medio para enfocar y desplegar la reflexión acerca de la relación entre su carrera académica y sus primeras prácticas profesionales. Esto significa, precisamente, reflexionar a través de la acción de ir transformándose en un profesional de la educación (Loughran y Russell, 1997). Esta estrategia de reflexión mediante un documento que los alumnos van reelaborando tiene larga data en el mundo anglosajón y se reporta en la literatura como "Filosofía Personal de la Educación" o "Declaración Pedagógica" (Personal Philosophy of Education 0 Teaching Statement) ${ }^{1}$.

\section{El ensayo de posición pedagógica}

En la tradición docente de Estados Unidos y Canadá es habitual que, para lograr graduarse, los futuros profesores deban redactar un Teaching Statement hacia el final de sus estudios. Este escrito de no más de dos páginas es tanto un requisito formal para recibir la certificación docente como un material solicitado por la mayoría de los empleadores del sistema educativo. Es decir, el alumno debe defender su ensayo tanto frente a sus profesores como a los directivos de las escuelas donde buscará trabajo (Montell, 2003a).

A partir de la revisión de la literatura, se encontraron tres tipos de instrumentos o modalidades a través de la cuales desarrollar el Teaching Statement: instrumentos orientados a la selección de personal con pocas preguntas, todas abiertas pero muy dirigidas para guiar el relato de los participantes (Eierman, 2008); instrumentos basados en imaginerías que estimulan el desarrollo de metáforas acerca de la educación (Chism, 1998); e instrumentos generados a partir de tarjetas con

\footnotetext{
${ }^{1}$ El teaching statement surgió como una recomendación de la Conferencia en Composición y Comunicación de los College de los Estados Unidos para que los futuros profesores contasen con un documento de síntesis de sus carreras, un hito que mostrara el grado de madurez profesional con la que los alumnos de educación abordarían sus futuras labores docentes. La presunción fundamental era que comprometerse en una reflexión explícita acerca de las propias ideas y creencias sobre la educación haría del maestro un profesional mucho más consciente de las decisiones que tendría que tomar en la sala de clases (Marini, 2015).
} 
conceptos pedagógicos clave para estimular la reflexión y luego articular un escrito (Beatty, Leigh y Lung Dean, 2009b). Los instrumentos utilizados para la selección de personal son los más ampliamente referidos en la literatura (Schönwetter, Sokal, Friesen y Taylor, 2002).

Para el estudio que se presenta en este artículo se acuñó la noción de "Ensayo de Posición Pedagógica" o EPP. En breve, el EPP se inspira en un Teaching Statement pero se distingue en dos aspectos concretos: en primer lugar, no se redacta hacia el final de la carrera, sino que su primera versión es intencionada durante el primer día de clases de los estudios para ser profesor, anticipándose que habrá al menos dos momentos para re-iterar o re-interpretar el mismo texto cada año. En segundo lugar, si bien el EPP puede cumplir con la función de oficiar como carta de presentación laboral, la expectativa inmediata es que sirva como testimonio vivo del desarrollo profesional de los profesores. Es decir, no está diseñado para dar una cierta impresión al empleador en una entrevista de trabajo sino más para registrar el itinerario pedagógico de los docentes.

En general, la literatura en el tema considera este tipo de estrategia como "un análisis racional, sistemático y crítico que se enfoca en los principales componentes que permiten definir una enseñanza y aprendizaje efectivos en una disciplina particular y/o contexto institucional" (Schönwetter et al.2002, p. 84). Su redacción suele abordar preguntas en cuatro ámbitos básicos: a) el significado y relación entre enseñar y aprender; b) las posibles metodologías de enseñanza y evaluación; c) el impacto de la experiencia de práctica profesional en las perspectivas que se tienen acerca de la educación; d) la relevancia social de la tarea educativa. No hay ningún desafío de la práctica docente que esté excluido como tema posible. Por ello, más que buscar soluciones únicas o definitivas, este ejercicio quiere visibilizar los distintos valores o perspectivas desde las cuales el docente enfrentará su tarea (Pratt, 2005).

Desde este punto de vista, el EPP podría concebirse como un proceso reflexivo que no se agota en la redacción de un documento, sino que el mayor aprendizaje para el profesor radica en la revisión periódica del mismo, proceso que sería incluso más importante que el acabado del texto (Beatty, Leigh y Lung Dean, 2009a). En concordancia, Ramsey y Fitzgibons (2005) precisan que un documento como este pretende abordar:

Quiénes somos [los profesores], en qué creemos, qué pensamos de los estudiantes, los materiales y el mundo que afectan significativamente lo que hacemos en la sala más allá del contenido del curso o estilo de enseñanza. Reconocer esto da mayor ímpetu para cuestionar continuamente y repensar quiénes somos en el mundo y cómo queremos que sean nuestros vínculos con los estudiantes y la enseñanza de la disciplina ( $p$. 345).

Finalmente, una estrategia como el EPP se hace cargo de la distancia entre la promesa de lograr profesores reflexivos, y la experiencia vital y profesional acumulada. $\mathrm{Si}$ bien los futuros profesores han pasado al menos doce años de su vida en el sistema escolar formal, al comenzar su formación profesional suelen declarar que desconocen lo fundamental de la labor docente (Russell, 2012). Por lo mismo, el EPP permitiría re-descubrir el valor de la experiencia de formación inicial docente como fuente inagotable de aprendizaje profesional (Marini, 2015).

\section{Contexto local}

Con una marcada influencia de Schön, el Ministerio de Educación de Chile ha ido formulando cada vez más explícitamente la necesidad de que el profesorado desarrolle sus capacidades de reflexión (Ávalos, 2002; 1998). Desde la Reforma Educacional de 1990 esta demanda se vio reflejada en los Objetivos Fundamentales y Contenidos Mínimos Obligatorios de la Educación Secundaria (Gobierno de Chile-MINEDUC, 1998), y en el Marco para la Buena Enseñanza que propone que los profesores "reflexionen consciente y sistemáticamente sobre su práctica y la reformulen, contribuyendo a garantizar una educación de calidad para todos los estudiantes" (Gobierno de Chile-MINEDUC, 2003, p. 10). Por su parte, los Nuevos Estándares Orientadores para Carreras de Pedagogía en Educación Secundaria postulan que:

El futuro profesor o profesora comprende que su desempeño profesional requiere de una dedicación a su aprendizaje continuo. Es capaz de analizar y reflexionar individual y colectivamente sobre su práctica pedagógica y sobre los resultados de aprendizaje de sus estudiantes. Está preparado para formular preguntas e hipótesis que le permitan recolectar y registrar sistemáticamente información para analizar y reflexionar individual y colectivamente sobre la práctica pedagógica propia, de otros docentes y del establecimiento educacional en donde realiza sus prácticas, y tomar decisiones pedagógicas (Gobierno de Chile-MINEDUC, 2012, p. 48) $)^{2}$.

\footnotetext{
${ }^{2}$ Durante la última década el Ministerio de Educación de Chile ha instituido una prueba voluntaria de diagnóstico para los egresados de carreras de pedagogía, llamada INICIA. Si bien esta prueba no posee carácter de certificación o habilitación -aun cuando hay quienes que prevén que 
Al momento de avanzar sobre las implicaciones prácticas de tales ideas, hay dos estrategias que se han consolidado en distintas instituciones formadoras chilenas como las más utilizadas para favorecer la reflexión de los futuros profesores: la bitácora y el portafolios.

La bitácora se inspira en el cuaderno de navegación que lleva el capitán de un barco y cumple con objetivos similares. Aquí el docente en formación va recabando progresivamente los hechos más notables de su práctica profesional y tiene la oportunidad de incorporar referencias a los cursos académicos que realiza en ese momento. La bitácora se caracteriza además por facilitar el análisis acumulativo de un hecho concreto en la experiencia del futuro profesor, permitiendo revisar el registro histórico de sus distintas implicancias (Inalef, Guerra, Aros y Scheihing, 2010) ${ }^{3}$.

El portafolios es literalmente la carpeta en la que los artistas llevan lo más notable de su trabajo (Seldin, 1997). En el ámbito de la formación inicial el portafolios está constituido por aquellos materiales que el mismo profesor ha seleccionado como lo más representativo de su labor, y a los que puede recurrir para fines de autoevaluación, desarrollo personal o de discusión pedagógica (Fernández March, 2004). Como la bitácora, la selección de la información depende del criterio del profesor. A diferencia de esta, su desarrollo no es necesariamente lineal, sino que puede ser recursivo y ahondar sobre un mismo tema, o constar de materiales distanciados en el tiempo entre sí.

Al comparar las características generales de las bitácoras y los portafolios con el EPP se puede afirmar lo siguiente:

El EPP promueve el análisis recursivo sobre el vínculo entre la propia experiencia, los cursos académicos y la práctica profesional. EI EPP no solo tematiza logros o hechos significativos, sino que da lugar a la exploración de los puntos ciegos, inconsistencias o tensiones que puedan surgir durante la práctica profesional. Si bien el EPP comienza a utilizarse durante la formación inicial, se busca extender su práctica a lo largo de la carrera de los profesores. Por el tipo de preguntas guías que el EPP propone, este se constituye como una evidencia del sentido pedagógico que el profesor encarna. El EPP promueve la mejora de la práctica docente por medio de la interrelación constante entre la teoría y la práctica, constituyéndose en un ejercicio de reflexión-acción.

\section{Objetivos}

Teniendo en cuenta el potencial impacto que el EPP podría tener en la formación inicial de profesores, el presente estudio se propone como objetivo general evaluar la progresión del EPP a lo largo de la trayectoria de formación de dichos estudiantes.

Además, la propuesta se orientó por los siguientes objetivos específicos:

1. Diseñar las intervenciones y preguntas orientadoras para el EPP.

2. Elaborar los criterios de análisis del EPP.

3. Implementar la redacción de tres versiones del EPP con estudiantes de un Programa anual de Pedagogía en Educación Media.

4. Indagar en las experiencias que se ponen en juego al escribir el EPP a través de entrevistas personales que discutan la transformación del mismo.

\section{Metodología Participantes}

Esta intervención educativa se realizó en el marco del Programa de Formación Pedagógica en Educación Media, de la Facultad de Educación de la Pontificia Universidad Católica de Chile ${ }^{4}$. Dicho Programa dura un año lectivo y está dirigido a licenciados en diferentes disciplinas. El Programa ofrece cursos generales para todos los estudiantes y un curso de didáctica de la disciplina específica. A la vez, tiene como eje una práctica anual que busca ser la instancia integradora de los diversos cursos. El primer semestre se denomina Práctica Inicial; allí los estudiantes observan clases de su disciplina en la escuela, colaboran con el profesor responsable, y culminan con la realización de una subunidad pedagógica de al menos 6 horas. El segundo semestre se denomina Práctica Profesional; la labor demandada es la enseñanza independiente de al menos 40 horas.

De un total de 89 matriculados, la muestra seleccionada fue de 47 estudiantes que firmaron los respectivos

esto suceda en el corto plazo a raíz de la Nueva Ley de Carrera Docente-, es importante destacar que uno de los ítems que evalúa INICIA es la capacidad de reflexión docente a partir de la redacción de un ensayo.

${ }^{3} \mathrm{Al}$ día de hoy, existe un desplazamiento desde el soporte en papel hacia el digital evidenciado por el creciente uso de Blogs, lo que ha permitido el desarrollo novedoso de bitácoras de construcción comunitaria.

${ }^{4}$ http://educacion.uc.cl/2015-01-08-21-56-30/programa-de-formacion-pedagogica 
consentimientos informados para participar en la intervención. La primera aplicación del instrumento fue durante la semana de introducción al Programa y participaron 47 estudiantes, el segundo fue después de haber terminado su Práctica Inicial y participaron 23 de los 47 estudiantes que redactaron la primera versión, y el tercer momento fue una vez finalizada la Práctica Profesional donde participaron 11 estudiantes que habían redactado las dos versiones anteriores (ver tabla 1). La disminución en la cantidad de sujetos puede obedecer al carácter voluntario de esta instancia, su implementación en un contexto extracurricular y/o a la disponibilidad real de los estudiantes durante un año de trabajo intenso.

Tabla 1: Caracterización de la muestra en cada aplicación

\begin{tabular}{|c|c|c|c|}
\hline & Aplicación 1 & Aplicación 2 & Aplicación 3 \\
\hline $\mathrm{N}$ & 47 & 23 & 11 \\
\hline \multirow{2}{*}{ Sexo } & 31 mujeres & 16 mujeres & 6 mujeres \\
& 16 hombres & 7 hombres & 5 hombres \\
\hline \multirow{3}{*}{ Especialidades } & $41 \%$ humanista & $57 \%$ humanista & $55 \%$ humanista \\
& $33 \%$ científico-matemática & $30 \%$ científico-matemática & $27 \%$ científico-matemática \\
& $26 \%$ artística & $13 \%$ artística & $18 \%$ artística \\
\hline
\end{tabular}

Teniendo en cuenta la literatura revisada, se optó adaptar un instrumento inspirado en aquellos utilizados para la selección de personal (Schönwetter et al., 2002). Este consiste en una serie de cuatro preguntas: a) ¿Cuál es para ti la relevancia social de la educación?; b) ¿Hay en tu historia alguna persona o experiencia que haya tenido una influencia especial en tu forma de ser profesor, que haya marcado tu visión sobre la enseñanza?; c) ¿Cómo describirías tu estilo de enseñanza (si no has tenido oportunidad de enseñar ¿cómo imaginas tu estilo?) y por qué piensas que es efectivo?; d) ¿Qué significa enseñar y aprender y cuál es su relación? Dichas preguntas se presentan a los estudiantes antes, durante y al finalizar su Programa de Formación, solicitándoles cada vez tener en cuenta las relaciones emergentes entre los cursos, prácticas y experiencias vividas.

La escala de apreciación utilizada (Tabla 2) es una adaptación de la rúbrica de evaluación de documentos semejantes al EPP creada por Schönwetter et al. (2002), la cual se modificó a partir de los objetivos de la intervención. A cada indicador se le asignó un puntaje de 0 a 4 (Ver Tabla 2).

Tabla 2: Descripción de puntajes asignados a cada indicador

\begin{tabular}{|c|c|l|}
\hline 0 & Insuficiente & $\begin{array}{l}\text { No hay indicios del indicador, el discurso del estudiante se centra en ołros } \\
\text { aspectos. }\end{array}$ \\
\hline 1 & Suficiente & $\begin{array}{l}\text { El indicador está implícito, por ejemplo, se alude indirectamente a la } \\
\text { relación enseñanza - aprendizaje: "los maestros y alumnos tienen que } \\
\text { hacer bien su trabajo". }\end{array}$ \\
\hline 2 & Bueno & $\begin{array}{l}\text { El indicador está presente de un modo literal pero no se profundiza, por } \\
\text { ejemplo, se explicita la relación enseñanza - aprendizaje: "es fundamental } \\
\text { que el docente enseñe a los alumnos y estos se muestren receptivos". }\end{array}$ \\
\hline 3 & Muy Bueno & $\begin{array}{l}\text { El indicador se declara y argumenta. Por ejemplo, se problematiza la } \\
\text { relación enseñanza - aprendizaje: "como profesor tengo que enseñar } \\
\text { de modo que los alumnos sean capaces de comprender el tema desde el } \\
\text { contexto de sus diversas experiencias". }\end{array}$ \\
\hline 4 & $\begin{array}{l}\text { El indicador se discute mediante ejemplos de la práctica profesional, } \\
\text { referencias académicas que avalan la discusión y/o tradiciones de la propia } \\
\text { disciplina (Ver ejemplos en Tabla 3). }\end{array}$ \\
\hline
\end{tabular}


Dada la complejidad de la escala y la necesidad de ajustarla en función de las características de los escritos de los participantes, todos los ensayos fueron codificados por dos evaluadores, se discutieron en el equipo las discrepancias y se llegó a un total acuerdo en cada una. Cada discusión permitió ajustar la escala de tal manera que los criterios de evaluación fueron ganando en especificidad.

Tabla 3: Escala de apreciación con ejemplos sugeridos para el nivel superior

\begin{tabular}{|c|c|c|c|}
\hline Dimensión & Descripción & Indicador & Ejemplos \\
\hline \multirow{6}{*}{\begin{tabular}{c|} 
A. Definición \\
de enseñanza y \\
aprendizaje
\end{tabular}} & \multirow{6}{*}{$\begin{array}{l}\text { Se definen y discuten } \\
\text { los términos enseñanza } \\
\text { y aprendizaje y su } \\
\text { relación, basándose } \\
\text { en literatura, en las } \\
\text { propias experiencias } \\
\text { o en ejemplos } \\
\text { apropiados. }\end{array}$} & $\begin{array}{l}\text { A.1. Define los términos enseñanza } \\
\text { y aprendizaje y discute su punto de } \\
\text { vista personal }\end{array}$ & $\begin{array}{l}\text { "Enseñar es dar luz, aprender es dar a luz; mi mamá trabajó } \\
\text { toda su vida de matrona y me doy cuenta que su trabajo es } \\
\text { una especie de metáfora del mío como profesora" }\end{array}$ \\
\hline & & $\begin{array}{l}\text { A.2. Explicita la relación entre } \\
\text { enseñanza y aprendizaje }\end{array}$ & $\begin{array}{l}\text { "Si enseñar implica generar las condiciones de experiencia } \\
\text { que hagan posible el aprendizaje como decía Dewey, } \\
\text { entonces aprender supone estar dispuesto a aprovechar } \\
\text { tales condiciones" }\end{array}$ \\
\hline & & $\begin{array}{l}\text { A.3. Sustenta con base en el } \\
\text { conocimiento profundo de la } \\
\text { literatura } y / 0 \text { cursos del Programa }\end{array}$ & $\begin{array}{l}\text { "Desde el curso "Gestión de aulas heterogéneas" y } \\
\text { "Evaluación" pude darme cuenta que respetar distintos } \\
\text { modos de conocer en los alumnos supone considerar } \\
\text { distintas estrategias de evaluación para ellos" }\end{array}$ \\
\hline & & $\begin{array}{l}\text { A.4. Incluye ejemplos variados } \\
\text { y apropiados sobre la propia } \\
\text { experiencia }\end{array}$ & $\begin{array}{l}\text { "Siempre he visto como mucha gente se aterra con solo } \\
\text { escuchar las palabras bioquímica, química o biología, o } \\
\text { sencillamente siente repudio hacia estas disciplinas" }\end{array}$ \\
\hline & & $\begin{array}{l}\text { A.5. Reflexiona sobre los ejemplos } \\
\text { presentados }\end{array}$ & $\begin{array}{l}\text { "Mis experiencias también me llevaron a ser estricta } \\
\text { y esperar que los alumnos cumplan con sus deberes y } \\
\text { las normas se acaten, pues siento que la disciplina y un } \\
\text { ambiente grato no son excluyentes" }\end{array}$ \\
\hline & & $\begin{array}{l}\text { A.6. Vincula su definición de } \\
\text { enseñanza y aprendizaje con su } \\
\text { experiencia de práctica }\end{array}$ & $\begin{array}{l}\text { "Sabía la fórmula pero no la veía en la realidad... Hasta } \\
\text { que le pregunté ¿Por qué no nos imaginamos el volcán } \\
\text { Villarica como dos triángulos rectángulos?" }\end{array}$ \\
\hline \multirow{5}{*}{$\begin{array}{l}\text { B. Metodología y } \\
\text { evaluación de la } \\
\text { enseñanza }\end{array}$} & \multirow{5}{*}{$\begin{array}{c}\text { Se evidencia } \\
\text { conocimiento acerca } \\
\text { de una variedad } \\
\text { de metodologías } \\
\text { de enseñanza y de } \\
\text { evaluación basadas } \\
\text { en conocimiento } \\
\text { de la literatura, en } \\
\text { características de } \\
\text { la disciplina y en } \\
\text { características del } \\
\text { aprendiz. }\end{array}$} & $\begin{array}{l}\text { B.1. Fundamenta en profundo } \\
\text { conocimiento de la literatura } y / 0 \\
\text { cursos del Programa }\end{array}$ & $\begin{array}{l}\text { "...por eso diseñé una evaluación que permita dar cuenta } \\
\text { si el alumno es capaz de aplicar lo aprendido a situaciones } \\
\text { fuera de la sala de clases. El aporte de Bransford con su } \\
\text { discusión de la transferencia me parece clarísimo" }\end{array}$ \\
\hline & & $\begin{array}{l}\text { B.2. Fundamenta en características } \\
\text { del aprendiz específicas a cada } \\
\text { disciplina }\end{array}$ & $\begin{array}{l}\text { "Siempre tengo en cuenta la necesidad de quitarle ese } \\
\text { miedo o rechazo a los alumnos hacia estas ciencias, sobre } \\
\text { todo a la química, que es a la que me dedicaré finalmente" }\end{array}$ \\
\hline & & $\begin{array}{l}\text { B.3. Fundamenta en características } \\
\text { del aprendiz específicas a su etapa } \\
\text { de desarrollo }\end{array}$ & $\begin{array}{l}\text { "Los dos talleres de filosofía me desafiaron mucho. Una cosa } \\
\text { es enseñar Platón a jóvenes de } 17 \text { años y otra es discutir } \\
\text { acerca de la justicia con niños de } 10 \text { años que todavía no } \\
\text { llegan a la etapa formal abstracta que plantea Piaget" }\end{array}$ \\
\hline & & $\begin{array}{l}\text { B.4. Da cuenta de su habilidad } \\
\text { para usar variadas estrategias de } \\
\text { enseñanza y evaluación }\end{array}$ & $\begin{array}{l}\text { "Me sorprendió que se sorprendieran por la autoevaluación. } \\
\text { Estaban acostumbrados a controles con preguntas cerradas } \\
\text { y abiertas pero la pregunta por cómo evaluarías to } \\
\text { aprendizaje los descolocó" }\end{array}$ \\
\hline & & $\begin{array}{l}\text { B.5. Muestra congruencia entre } \\
\text { estrategias mencionadas y las } \\
\text { definiciones de enseñanza y } \\
\text { aprendizaje }\end{array}$ & $\begin{array}{l}\text { "Esto es clase de filosofía. Yo puedo modelar el aprendizaje } \\
\text { de ciertos argumentos según mi propia experiencia pero la } \\
\text { capacidad crítica supone un compromiso con la pregunta por } \\
\text { parte del alumno" }\end{array}$ \\
\hline
\end{tabular}




\begin{tabular}{|c|c|c|c|}
\hline & & $\begin{array}{l}\text { B.6. Muestra congruencia entre } \\
\text { estrategias mencionadas y su } \\
\text { comprensión de la relación } \\
\text { profesor-alumno }\end{array}$ & $\begin{array}{l}\text { "Todo esto me ha llevado a comportarme en clases como } \\
\text { una profesora amigable, que bajo ninguna circunstancia } \\
\text { desea inspirar terror en los alumnos" }\end{array}$ \\
\hline & & $\begin{array}{l}\text { B.7. Vincula las metodologías y } \\
\text { evaluación de la enseñanza con su } \\
\text { experiencia de práctica }\end{array}$ & $\begin{array}{l}\text { "¿Quién iba a decir que yo iba a construir un cuestionario } \\
\text { de opción múltiple? Siempre le tuve mala idea a los } \\
\text { instrumentos estandarizados. Pero en el contexto de la } \\
\text { práctica me vino muy bien para saber de dónde estaba } \\
\text { partiendo con los alumnos" }\end{array}$ \\
\hline \multirow{3}{*}{$\begin{array}{l}\text { C. Contexto } \\
\text { personal de } \\
\text { enseñanza }\end{array}$} & \multirow{3}{*}{$\begin{array}{l}\text { Se evidencia un uso de } \\
\text { lenguaje disciplinar } \\
\text { a través del cual se } \\
\text { expresa conocimiento } \\
\text { de un contexto } \\
\text { institucional espeć́fico } \\
\text { y se articula con } \\
\text { cómo su visión de la } \\
\text { enseñanza se ajusta a } \\
\text { ese contexto }\end{array}$} & C.1. Usa lenguaje disciplinar & $\begin{array}{l}\text { "Además, la materia de cualquier tipo está formada por } \\
\text { partículas que la química ha podido explicar. Incluso, en } \\
\text { cosas más cotidianas como cocinar, también la química está } \\
\text { involucrada" }\end{array}$ \\
\hline & & $\begin{array}{l}\text { C.2. Demuestra con claridad un } \\
\text { amplio conocimiento del clima } \\
\text { institucional específico en que se } \\
\text { inserta. }\end{array}$ & $\begin{array}{l}\text { "Justo me tocaba el tema del mal en la unidad de ética y } \\
\text { sabía que la mamá de un alumno estaba enferma. Conversé } \\
\text { con el profesor jefe y el coordinador de convivencia escolar, } \\
\text { eso me ayudó a sentirme más segura en cómo plantear el } \\
\text { tema sin herir a nadie" }\end{array}$ \\
\hline & & $\begin{array}{l}\text { C.3. Refleja una postura frente } \\
\text { a la relación entre expectativas } \\
\text { personales y demandas } \\
\text { institucionales. }\end{array}$ & $\begin{array}{l}\text { "La UTP [responsable pedagógica] está encima de todo, } \\
\text { quizás un poco controladora. Yo esperaría trabajar en un } \\
\text { ambiente de mayor confianza pero entiendo que como } \\
\text { practicante todavía no me conocen. Espero ganarme su } \\
\text { confianza en el futuro" }\end{array}$ \\
\hline \multirow{5}{*}{$\begin{array}{l}\text { D. Organización } \\
\text { textual }\end{array}$} & \multirow{5}{*}{$\begin{array}{l}\text { El escrito se encuadra } \\
\text { a través de una } \\
\text { metáfora ilustrativa o } \\
\text { un incidente crítico que } \\
\text { facilita la articulación } \\
\text { de las distintas } \\
\text { partes. Se observa } \\
\text { una progresión } \\
\text { coherente entre las } \\
\text { partes y evidencias de } \\
\text { pensamiento crítico y } \\
\text { ejemplos que articulan } \\
\text { el escrito. }\end{array}$} & $\begin{array}{l}\text { D.1. Enmarca el EPP dentro de una } \\
\text { metáfora ilustrativa o incidente } \\
\text { crítico }\end{array}$ & \multirow{2}{*}{$\begin{array}{l}\text { "Mi padre tuvo que salir a trabajar en el campo cuando era } \\
\text { niño para ayudar a mi abuelo. No pudo ir ni a la Básica. Hoy } \\
\text { día todavía no lee ni escribe bien. Yo voy a ser profesora } \\
\text { para ayudar a que los niños aprendan a leer desde pequeños } \\
\text { y ayuden a sus familias" }\end{array}$} \\
\hline & & $\begin{array}{l}\text { D.2. La metáfora o incidente crítico } \\
\text { demuestra vínculos coherentes con } \\
\text { los diversos componentes del EPP }\end{array}$ & \\
\hline & & $\begin{array}{l}\text { D.3. Muestra congruencia entre los } \\
\text { componentes del EPP }\end{array}$ & \multirow{3}{*}{$\begin{array}{l}\text { Estos indicadores se deducen, implican o contienen al } \\
\text { relacionar dos o más de los indicadores anteriores }\end{array}$} \\
\hline & & $\begin{array}{l}\text { D.4. Evidencia la capacidad de } \\
\text { pensamiento crítico y reflexivo }\end{array}$ & \\
\hline & & $\begin{array}{l}\text { D.5. Muestra capacidad de } \\
\text { dar ejemplos específicos en la } \\
\text { articulación de sus creencias } y / 0 \\
\text { acciones } y / 0 \text { metas }\end{array}$ & \\
\hline
\end{tabular}

\section{Entrevista}

Finalmente, de entre quienes realizaron los tres ensayos se entrevistó a los 5 estudiantes que respondieron a la invitación con el fin de indagar su apreciación sobre el EPP. Se plantearon las siguientes preguntas:

I. ¿Cuáles fueron las fuentes con las que te conectaste para escribir esa primera versión?

II. ¿Cuál fue la experiencia, momento de la Práctica Profesional y/o curso del Programa que más te impactó este año?

III. ¿Te parece que tu posición pedagógica cambió a lo largo de este año? ¿Por qué? ¿Dónde? ¿En qué sentido?

IV. ¿Cómo evalúas este proceso de redactar tu posición pedagógica, y reescribirla dos veces durante el año? 


\section{Procedimiento}

El primer escrito fue desarrollado por los estudiantes en el contexto de la primera semana de inducción al Programa. Con el fin de que esta versión recogiera sus experiencias educativas previas al ingreso al programa, se cauteló que los estudiantes no hubieran tenido ninguna participación en actividades curriculares formales. En esta instancia se presentó a los estudiantes el estudio, sus tareas y lo que se esperaba de ellos. Se leyó el consentimiento informado y cada estudiante pudo elegir si participar o no del estudio. Los participantes que disponían de computador elaboraron directamente su ensayo en formato digital, y el resto lo escribió de forma manual y luego fueron digitalizados por el equipo de investigación. La redacción del primer ensayo fue digitalizada a través de una transcripción a documento Word MS. Los siguientes dos ensayos se realizaron uno, una vez finalizada la práctica inicial y el otro, una vez finalizada la práctica profesional. Cada ensayo se realizó en una sala de computación donde cada estudiante dispuso de un computador y de su EPP digitalizado, recibiendo solamente la instrucción de revisar su escrito. Para facilitar la comparación y revisión de los ensayos, se utilizó la herramienta de control de cambios. El propósito de esta estrategia fue captar la diferencia entre los escritos y las dimensiones en que dichas diferencias se manifestaban.

\section{Análisis de datos}

A partir del análisis de los textos producidos por los estudiantes, se generó una base de datos con los puntajes acordados entre codificadores para cada uno de los indicadores de cada dimensión. Para la descripción cuantitativa de las variables (1) definición de enseñanza aprendizaje, 2) metodología y evaluación de la enseñanza, 3) contexto personal de enseñanza, 4) organización textual, se calculó el promedio de cada sujeto, desviaciones y frecuencia de los datos recolectados en cada dimensión en cada una de las tres aplicaciones. Para el análisis de los estadísticos del instrumento se utilizó el programa SPSS. Con los resultados de la primera aplicación se evaluó su consistencia interna mediante el estadístico alfa de Cronbach $(0,915)$, observándose niveles de consistencia aceptables para su uso. Para la comparación de los resultados en los tres momentos se usó el análisis no paramétrico de muestras relacionadas a través de una prueba de Wilcoxon. Para el análisis comparativo de los ensayos entre la primera y la segunda aplicación no se consideraron los indicadores A.3, A.6, B.1, B.7, C.2, C.3 (Ver Tabla 2) por estar relacionados con experiencias de práctica y de cursos que los estudiantes no habían tenido al iniciar el programa de formación.

Por su parte, con las entrevistas se hizo un análisis de contenidos que permitió establecer categorías a la base de los cambios percibidos por los propios participantes en sus respectivos ensayos.

\section{Resultados}

\section{Relaciones entre las dimensiones evaluadas}

Para explorar las relaciones entre las distintas dimensiones establecidas en el instrumento, se hizo un análisis de correlaciones bivariado, cuyos resultados se presentan en la tabla 3.

Se observan correlaciones significativas entre las dimensiones definidas teóricamente, pero lo suficientemente bajas como para indicar que cada una de ellas está midiendo aspectos distintos del ensayo.

Tabla 4: Correlación entre dimensiones de la tarea de generar explicaciones de calidad

\begin{tabular}{|l|c|c|c|c|}
\hline Dimensiones & A & B & C & D \\
\hline A. Definición de enseñanza y aprendizaje & - & $0.664^{* *}$ & $0.366^{*}$ & $0,793^{* *}$ \\
\hline B. Metodología y Evaluación & & - & $0.370^{*}$ & $0.598^{*}$ \\
\hline C. Contexto personal de enseñanza & & & - & $0.538^{*}$ \\
\hline D. Organización textual & & & & - \\
\hline \multicolumn{2}{|c|}{ Nota. $^{*}=p<0.01 ;$} \\
\end{tabular}




\section{Descriptivos de las tres aplicaciones}

Por otra parte, se calculó la media y la desviación estándar para cada uno de los indicadores y para el promedio de cada dimensión evaluada. En la Tabla 5 se presentan todos los datos con el fin de poder tener una perspectiva general de los resultados para cada una de las aplicaciones.

Tabla 5: Descriptivos de las tres aplicaciones

\begin{tabular}{|c|c|c|c|c|c|c|}
\hline \multirow[b]{2}{*}{ Dimensión/ Indicador } & \multicolumn{2}{|c|}{$\begin{array}{c}\text { Aplicación } 1 \\
n=47\end{array}$} & \multicolumn{2}{|c|}{$\begin{array}{c}\text { Aplicación } 1 \\
n=47\end{array}$} & \multicolumn{2}{|c|}{$\begin{array}{c}\text { Aplicación } 1 \\
n=47\end{array}$} \\
\hline & M & SD & M & SD & M & SD \\
\hline A. Definición de enseñanza- aprendizaje & 2.07 & 0.62 & 1.33 & 0.47 & 1.45 & 0.82 \\
\hline $\begin{array}{l}\text { A.1 Define los términos enseñanza y aprendizaje y discute su } \\
\text { punto de vista personal }\end{array}$ & 2.71 & 0.52 & 2.48 & 0.51 & 2.27 & 0.47 \\
\hline A.2 Explicita relación entre enseñanza y aprendizaje & 2.01 & 0.67 & 1.61 & 0.72 & 1.63 & 0.67 \\
\hline $\begin{array}{l}\text { A.3 Sustenta con base en el conocimiento profundo de la } \\
\text { literatura } y / 0 \text { cursos del Programa }\end{array}$ & - & - & 0.56 & 0.78 & 0.45 & 0.69 \\
\hline $\begin{array}{l}\text { A.4 Incluye ejemplos variados y apropiados sobre la propia } \\
\text { experiencia }\end{array}$ & 1.85 & 1.06 & 1.3 & 0.76 & 1.45 & 0.52 \\
\hline A.5 Reflexiona sobre los ejemplos presentados & 1.71 & 0.98 & 1.26 & 0.75 & 1.45 & 0.52 \\
\hline $\begin{array}{l}\text { A.6 Vincula su definición de enseñanza y aprendizaje con su } \\
\text { experiencia de práctica }\end{array}$ & - & - & 0.74 & 0.86 & 1.45 & 0.82 \\
\hline B. Metodología y evaluación de la enseñanza & 1.39 & 0.57 & 1.17 & 0.41 & 1.29 & 0.4 \\
\hline $\begin{array}{l}\text { B.1 Fundamenta en profundo conocimiento de la literatura } \\
\text { y/o cursos del Programa }\end{array}$ & - & - & 0.52 & 0.67 & 0.36 & 0.67 \\
\hline $\begin{array}{l}\text { B.2 Fundamenta en características del aprendiz específicas a } \\
\text { cada disciplina }\end{array}$ & 0.94 & 0.77 & 1.04 & 0.93 & 1.1 & 0.7 \\
\hline $\begin{array}{l}\text { B.3 Fundamenta en características del aprendiz específicas a } \\
\text { su etapa de desarrollo }\end{array}$ & 0.89 & 0.58 & 0.96 & 0.47 & 0.91 & 0.54 \\
\hline $\begin{array}{l}\text { B.4 Da cuenta de su habilidad para usar variadas estrategias } \\
\text { de enseñanza y evaluación }\end{array}$ & 1.68 & 0.77 & 1.78 & 0.56 & 1.91 & 0.54 \\
\hline $\begin{array}{l}\text { B.5 Muestra congruencia entre estrategias mencionadas y las } \\
\text { definiciones de enseñanza y aprendizaje }\end{array}$ & 1.68 & 0.67 & 1.39 & 0.66 & 1.73 & 0.47 \\
\hline $\begin{array}{l}\text { B.6 Muestra congruencia entre estrategias mencionadas y su } \\
\text { comprensión de la relación profesor-alumno }\end{array}$ & 1.78 & 0.62 & 1.83 & 0.58 & 2 & 0.53 \\
\hline $\begin{array}{l}\text { B.7 Vincula las metodologías y evaluación de la enseñanza } \\
\text { con su experiencia de práctica }\end{array}$ & - & - & 0.7 & 0.88 & 1 & 0.89 \\
\hline C. Contexto personal de enseñanza & 1.47 & 0.96 & 0.86 & 0.44 & 1.03 & 0.46 \\
\hline C.1 Usa lenguaje disciplinar & 1.47 & 0.96 & 2 & 0.64 & 2.1 & 0.3 \\
\hline $\begin{array}{l}\text { C.2 Demuestra con claridad un amplio conocimiento del } \\
\text { clima institucional espećítico en que se inserta. }\end{array}$ & - & - & 0.35 & 0.71 & 0.45 & 0.82 \\
\hline $\begin{array}{l}\text { C.3 Refleja una postura frente a la relación entre expectativas } \\
\text { personales y demandas institucionales. }\end{array}$ & - & - & 0.3 & 0.47 & 0.55 & 0.52 \\
\hline D. Organización textual & 2.09 & 0.52 & 1.77 & 0.45 & 1.73 & 0.4 \\
\hline $\begin{array}{l}\text { D.1 Enmarca el EPP dentro de una metáfora ilustrativa o } \\
\text { incidente crítico }\end{array}$ & 1.88 & 0.76 & 1.7 & 0.76 & 1.63 & 0.81 \\
\hline $\begin{array}{l}\text { D.2 La metáfora o incidente crítico demuestra vínculos } \\
\text { coherentes con los diversos componentes del EPP }\end{array}$ & 1.95 & 0.78 & 1.6 & 0.72 & 1.55 & 0.82 \\
\hline D.3 Muestra congruencia entre los componentes del EPP & 2.29 & 0.52 & 2.04 & 0.37 & 1.91 & 0.3 \\
\hline D.4 Evidencia la capacidad de pensamiento crítico y reflexivo & 2.39 & 0.57 & 2.17 & 0.49 & 2.1 & 0.54 \\
\hline $\begin{array}{l}\text { D.5 Muestra capacidad de dar ejemplos específicos en la } \\
\text { articulación de sus creencias } y / 0 \text { acciones } y / 0 \text { metas }\end{array}$ & 1.93 & 0.62 & 1.35 & 0.71 & 1.45 & 0.52 \\
\hline
\end{tabular}


Al iniciar el programa de formación en las dimensiones de definición de enseñanza y aprendizaje $(M=2.07)$ y organización textual $(\mathrm{M}=2.09)$ se observan los desempeños más altos de la muestra. Los indicadores en que se observa mayor logro son: define los términos enseñanza y aprendizaje y discute su punto de vista personal $(M=2.71)$ a nivel de definición de enseñanzaaprendizaje, y a nivel de organización textual, evidenciar la capacidad de pensamiento crítico y reflexivo $(\mathrm{M}=$ 2.39). La dimensión en que se observa el desempeño más bajo es en la metodología y evaluación de la enseñanza ( $M=1.39)$, específicamente en los indicadores relacionados con la fundamentación en características del aprendiz específicas a su etapa del desarrollo ( $M=$ 0.89) y específicas a la disciplina $(M=0.94)$.

Para la segunda aplicación, realizada una vez terminada la práctica inicial después de 16 semanas de formación, la dimensión con el promedio más alto es la organización textual $(\mathrm{M}=1.77)$ y la dimensión con el promedio más bajo es el contexto personal de enseñanza $(M=0.86)$. Los indicadores relacionados con sustentar el conocimiento profundo de la literatura y/o cursos del programa $(\mathrm{M}=0.52)$, demostrar con claridad un amplio conocimiento del clima institucional específico en que se inserta $(M=0.35)$ y reflejar una postura frente a la relación entre expectativas personales y demandas institucionales de los centros de práctica $(M=0.3)$ aparecen como los indicadores más bajos de esta segunda aplicación.

En la tercera aplicación, realizada una vez terminada la práctica profesional, la dimensión en que se observa el promedio más alto es la organización textual. Los indicadores con los promedios más altos para este grupo son: define los términos enseñanza y aprendizaje y discute su punto de vista personal $(\mathrm{M}=2.27)$, usa lenguaje disciplinar $(M=2.21)$ y evidencia la capacidad de pensamiento crítico y reflexivo $(M=2.21)$. La dimensión con el promedio más bajo es la consideración del contexto personal de enseñanza $(M=1.03)$ y los indicadores de menor desempeño son: fundamenta un profundo conocimiento de la literatura y/o cursos del Programa ( $\mathrm{M}=$ 0.36 ) y muestra con claridad un amplio conocimiento del clima institucional específico en que se inserta $(M=0.45)$.

En síntesis, en estas tres versiones aparece la Organización Textual como la dimensión con el mejor promedio observado y la dimensión Metodología y Evaluación, y Contexto Personal de Enseñanza como las dimensiones con el promedio observado más bajo. Dentro de Organización Textual, el indicador con el promedio observado más alto en los tres momentos aplicados es "evidencia pensamiento crítico y reflexivo" y el más bajo "muestra capacidad de dar ejemplos específicos en la articulación de sus creencias y/o acciones y/o metas".

El indicador que aparece con el promedio observado más alto en los tres ensayos es "define los términos enseñanza y aprendizaje y discute su punto de vista personal" de la dimensión Definición de Enseñanza y Aprendizaje. Por su parte, los indicadores que se observan con el promedio más bajo son tres: "fundamenta a partir de un profundo conocimiento de la literatura y/o cursos del Programa de Formación" de la dimensión Metodología y Evaluación de la Enseñanza; y "demuestra con claridad un amplio conocimiento del clima institucional específico en que se inserta" y "refleja una postura frente a la relación entre expectativas personales y demandas institucionales" de la dimensión Contexto Personal de Enseñanza.

\section{Análisis comparativo}

A continuación, se analizan los resultados en relación al objetivo de "evaluar la progresión del Ensayo de Posición Pedagógica a lo largo de la trayectoria de formación de los estudiantes". De acuerdo a los resultados descriptivos presentados en la tabla 6 , la dimensión que tuvo el mejor resultado en las tres aplicaciones fue la organización textual y la que tiene los promedios observados más bajos es la dimensión relacionada con la consideración del contexto personal en el ensayo.

Tabla 6: Promedios, desviaciones para la muestra en las tres aplicaciones

\begin{tabular}{|c|c|c|c|c|c|c|c|}
\hline & & \multicolumn{2}{|c|}{ Aplicación 1 } & \multicolumn{2}{c|}{ Aplicación 2 } & \multicolumn{2}{c|}{ Aplicación 3} \\
\hline Dimensión & $\mathrm{n}$ & $\mathrm{M}$ & $\mathrm{SD}$ & $\mathrm{M}$ & $\mathrm{SD}$ & $\mathrm{M}$ & SD \\
\hline $\begin{array}{c}\text { Definición enseñanza } \\
\text { aprendizaje }\end{array}$ & 11 & 2.11 & 0.55 & 1.33 & 0.4 & 1.45 & 0.47 \\
\hline $\begin{array}{c}\text { Metodología y } \\
\text { evaluación }\end{array}$ & 11 & 1.62 & 0.49 & 1.23 & 0.32 & 1.29 & 0.4 \\
\hline Contexto personal & 11 & 1.86 & 0.92 & 0.85 & 0.38 & 1.03 & 0.46 \\
\hline Organización textual & 11 & 2.12 & 0.55 & 1.83 & 0.28 & 1.72 & 0.4 \\
\hline
\end{tabular}


En términos descriptivos, se observa que hay una disminución de todos los promedios entre la primera y la segunda aplicación. Dado que para la primera instancia no se analizaron los indicadores que implicaban establecer una relación con la experiencia en el programa, no es posible realizar comparaciones entre estos dos momentos a nivel cuantitativo. Sin embargo, si se puede comparar la segunda aplicación con la tercera porque en este caso se analizaron todos los indicadores en ambas instancias. A nivel descriptivo se observa un aumento en todas las dimensiones, a excepción de la organización textual. Para contrastar si las diferencias observadas son significativas se aplicó una prueba de Wilcoxon, un análisis no paramétrico de muestras relacionadas. No se observan diferencias significativas entre los promedios observados. El único indicador que presenta diferencias significativas a favor de la tercera aplicación es: "vincula su definición de enseñanza y aprendizaje con su experiencia de práctica" (Wilcoxon, $p=0.034)$.

\section{Entrevistas}

Las entrevistas permiten entrever que, al momento de redactar la primera versión del EPP, cuatro de los cinco estudiantes recurren explícitamente a experiencias de vida y solo tangencialmente a algún aspecto de su formación profesional. Las fuentes que inspiran esta primera redacción son del tipo "En algún momento específico yo había hecho clases en un contexto desventajado" (I, 2), "me acordé de mi abuelita que era profesora normalista", (II, 3) "la experiencia que tuve en el colegio" (III, 2), "mi intención primera iba a ser y lo sigue siendo crear y poder vivir haciendo arte" (IV, 6).

Por otra parte, existe consenso unánime al considerar a la Práctica Profesional como el curso con mayor impacto en el Programa de Formación. Tres de los entrevistados destacaron además a la Didáctica de su especialidad (I, 4; II, 44; IV, 24).

Respecto del cambio en el propio EPP a lo largo del año, los cinco entrevistados indican que hay dos formas de cambio: entre el primer y segundo ensayo las prácticas profesionales impactan radicalmente en la concepción de la tarea docente; entre el segundo y tercer ensayo se profundizan las impresiones generadas a partir de las prácticas profesionales: "En el primer ensayo escribí que no me importaba tanto que el profesor dominara los contenidos sino que más me importaba su fortaleza y solidez valórica pero después en el segundo me di cuenta de que sí importa también, porque si uno no tiene el dominio de los contenidos claros como que eso es mala comunicación de la disciplina, también es un error grave que es algo de lo que tomé conciencia después de haber observado un semestre de clases" (II, 22). "... entre el primero y el segundo yo creo que esa es la gran diferencia. Entre el segundo y el tercero yo creo que es enfocarse un poco más en las ideas, es darle vuelta a lo que había escrito antes, sin cambiarlo mucho, sin cambiar la esencia de donde iba antes, sino que ahondar un poco más, describir un poco más las situaciones, describir un poco más los argumentos" (III, 51).

En cuanto a la evaluación del proceso de redactar el EPP a comienzos de año, y revisarlo a mediados y fin de año, los cinco entrevistados señalan lo positivo de la experiencia, y critican la falta de tiempo que los docentes en formación disponen para reflexionar acerca de su práctica. Además se recomienda la inclusión formal del EPP en un curso del Programa: "Encuentro que este ejercicio, yo lo encontré buenísimo, ojalá fuera obligatorio, porque creo que fue muy importante para mí, o sea, yo pensaba esto, lo pensaba y lo sabía, pero creo uno al escribir las cosas, [...] se hacen realidad y como que uno las tiene más presentes, y de verdad que yo noto que mi perspectiva fue cambiando, fue creciendo, se enriqueció el ensayo, quizás no creció todo lo que podría haber crecido si lo hubiese hecho en mi casa en una semana yo sola, pero de todas maneras fue una instancia para reflexionar, y de todas maneras creo que eso es algo que falta en nuestro programa, porque es de un año muy corto, muy rápido y las instancias de reflexión son muy pocas” (II, 30).“... el primer ensayo fue la primera semana, me parece muy bien, pero los otros dos colocarlos en un curso y que se discutan [...] en el Programa están todos siempre corriendo, darse un tiempo, revisemos un poquito esto también es muy importante, es ver cómo estoy evolucionando, cuáles son tus proyecciones, date cuenta, has cambiado, y si no has cambiado entonces partiste muy bien y si no preocúpate porque hay algo de lo que no te has dado cuenta, o de verdad no te ha servido esto" (III, 64).

\section{Discusión}

Al comparar los cambios observados en los estudiantes que participaron de las tres instancias de reescritura de los ensayos, no se observan diferencias significativas entre la segunda y la tercera aplicación. A primera vista parece contradictorio que un profesor con nueve meses de formación disciplinaria y pedagógica no tenga un relato significativamente más articulado, preciso y/o profundo a la hora de explicar, por ejemplo, cuál es su Definición de Enseñanza y Aprendizaje, que un licenciado en su primer semestre de formación, en que no ha tenido la experiencia de hacerse cargo de la enseñanza de un curso en forma autónoma. Esto se podría explicar de diferentes formas:

Por una parte, como surgió en distintas entrevistas, al comienzo de año la mayoría de los futuros profesores tiene una perspectiva idealizada de la labor docente. Por 
ejemplo: "Al principio uno entra recontra idealizado, en el sentido de la experiencia propia, pero sin estar inmerso en el sistema" (I, 48); "En el primer ensayo escribí que no me importaba tanto que el profesor dominara los contenidos, sino que más me importaba su fortaleza y solidez valórica" (II, 22). Esto estaría en concordancia con la apreciación de Russell (2012) respecto de que al inicio de sus carreras los alumnos de pedagogía suelen declarar que carecen de experiencia docente, por lo cual recurren a lugares comunes y/o a expresiones de deseabilidad social que dan cuenta de buenas intenciones más que de un conocimiento profesional.

Por otra parte, la experiencia en los centros de práctica es ocasión para que los estudiantes de pedagogía aterricen sus posturas. Como afirma Zeichner (1996), solo quienes "deambulan en las pantanosas arenas de la práctica" son capaces de reexaminar sus presunciones $y$, eventualmente, decidir cómo llevar adelante su trabajo de modos nuevos. Las entrevistas dan cuenta de esta posibilidad: "Yo salgo [de la universidad] con un estándar muy alto y creí que todos tenían el mismo [...] me he dado cuenta que no" (III, 30); "No pasaba mucho [en el Centro de Prácticas], entonces enfrentarme a esa realidad fue muy desafiante y yo me dije quiero hacer algo acá, quiero que pasen cosas" ( $\mathrm{V}, 22)$; "[pero] si uno no tiene el dominio de los contenidos claros como que eso es mala comunicación de la disciplina, también es un error grave que es algo de lo que tomé conciencia después de haber observado un semestre de clases" (II, 22).

Si bien a la luz de Schön (1983) los dos argumentos mencionados parecen un tanto obvios, ya que es esperable que la experiencia profesional proporcione una dosis de realismo, al comparar la segunda aplicación del EPP con la tercera, si bien no se observaron diferencias significativas, se notó un incremento en las mismas dimensiones: Definición de Enseñanza y Aprendizaje, Metodología y Evaluación de la Enseñanza y Contexto Personal de Enseñanza. Esto es relevante ya que podría indicar una mayor distancia experiencial entre el primer ensayo (en el que los alumnos carecen de experiencia docente) y el segundo (en el que comienzan con su práctica inicial) que entre el segundo y el tercero (en el que parecen ganar reflexión desde su propia práctica). Las entrevistas aluden a esta situación, por ejemplo: "Entre el primero y el segundo yo creo que esa es la gran diferencia. Entre el segundo y tercero yo creo que es enfocarse un poco más en las ideas, es darle vueltas a lo que había escrito antes, sin cambiarlo mucho, sin cambiar la esencia de donde iba antes, sino que ahondar un poco más" (IV, 17).
Esta suposición está alineada con lo señalado por Marini (2015) y Schonwetter et al. (2002) respecto del potencial del EPP para favorecer una exploración crítica y constructiva de la convergencia entre práctica profesional, cursos de formación universitaria y experiencia vital. Dicho de otro modo, una vez que el alumno constata por sí mismo la distancia entre sus presunciones acerca de la tarea de educar y el carácter multifacético de la práctica docente (Beatty et al., 2009a y 2009b), tiene sentido asumir que se encontrará profesionalmente dispuesto a re-examinar las bases y principios que justifican sus decisiones pedagógicas.

\section{Conclusión}

En tanto estrategia de formación continua, el EPP puede contribuir a que cada profesor identifique explícitamente los hitos de su carrera a través de los distintos tipos de cambio que va experimentando su texto. De este modo, el circuito siempre complejo entre contexto laboral, condiciones de vida personal y proyección profesional se manifestaría dejando ver el modo en que los profesionales de la educación maduran en su ejercicio, permitiendo evaluar qué dimensiones de su trabajo contribuyen u obstaculizan con su proyecto de vida. En pocas palabras, como una hoja de ruta viva y consciente, el EPP permitiría revalorizar la experiencia del profesor como nexo entre su formación universitaria y la práctica profesional.

Como orientaciones futuras, postulamos que el ejercicio propuesto por el EPP contribuye a la reflexión explícita acerca de las propias ideas y creencias sobre la educación. Es por esto que los participantes sugieren que se formalice esta instancia a través de su incorporación como una actividad de curso, por ejemplo, en los Talleres de Práctica. Si se valora el ejercicio de escribir y reescribir en forma personal, podría ser enriquecedor el que los estudiantes compartieran sus escritos entre ellos.

Además, sería valioso considerar la reescritura de este ensayo durante el primer año de ejercicio profesional. Convocar a los egresados a revisar el EPP que escribieron al terminar su formación inicial después de un año o ciclo de enseñanza completo podría representar una instancia de reflexión para ellos y una oportunidad de retroalimentación para los programas de formación inicial docente. Esta iniciativa podría constituirse en una herramienta relevante para vincularse con los egresados desde el sentido que la profesión va teniendo para cada uno. 


\section{Referencias}

Ávalos, B. (2002). Profesores para Chile: Historia de un proyecto. Santiago de Chile: MINEDUC

Ávalos, B. (1998). School-based teacher development the experience of teacher professional groups in secondary schools in Chile. Teaching and teacher education, 14 (3), 257-271. DOI:/10.1016/S0742051X(97)00040-1

Beatty, J.; Leigh, J. y Dean, K.L. (2009a). Finding Our Roots: An Exercise For Creating a Personal Teaching Philosophy Statement. Journal of Management Education, 33 (1), 115-130. DOI: /10.1177/1052562907310642

Beatty, J.; Leigh, J. y Dean, K.L. (2009b). Philosophy Rediscovered: Exploring the Connections between Teaching Philosophies, Educational Philosophies, and Philosophy. Journal of Management Education, 33 (1), 99-114. DOI: 10.1177/1052562907310557

Chism, N.V.N. (1998). Developing a philosophy of teaching statement. Essays on Teaching Excellence: Toward the Best in the Academy, 9 (3), 1-3. Disponible en: http://ucat.osu.edu/wordpress/ assets/V9-N3-Chism.pdf

Eierman, R. (2008). The Teaching Philosophy Statement: Purposes and Organizational Structure. Chemical Education Today, 85 (3), 336-339. DOI: 10.1021/ ed085p336

Fernández March, A. (2004). El portafolio docente como estrategia formativa y de desarrollo profesional. Educar (33), 127-142

Inalef, K.; Guerra, J.; Aros, C. y Scheihing, E. (2010). Desarrollo de una bitácora virtual para el registro de interacciones en clases presenciales. Ponencia presentada en el " $\mathrm{V}$ Congreso Iberoamericano de Informática Educativa", Santiago de Chile. Disponible en: http://www.tise.cl/volumen6/ TISE2010/Documento84.pdf

Kretchmar, K., Zeichner, K. (2016). Teacher prep 3.0: a vision for teacher education to impact social transformation. Journal of Education for Teaching, 42 (4),417-433.DOI:10.1080/02607476.2016.1215550

Loughran, J. y Russell, T. (1997). Meeting student teachers on their own terms: Experience precedes understanding. En Virginia Richardson (coord.). Constructivist teacher education: Building a world of new understandings, 164-181. London: Falmer Press

Gobierno de Chile-MINEDUC (2012). Estándares Orientadores para Carreras de Pedagogía en Educación Media. Santiago de Chile: Autor

Gobierno de Chile-MINEDUC (2003). Marco para la Buena Enseñanza. Santiago de Chile: Autor

Gobierno de Chile-MINEDUC (1998). Objetivos Fundamentales y Contenidos Mínimos Obligatorios de la Educación Básica y Media. Santiago de Chile: Autor

Marini, G. (2015). Michel Montaigne como Formador de Profesores: la Necesidad de Ensayar la Propia Posición Pedagógica. Trans/Form/Ação 38 (3), 117132. DOI:/10.1590/S0101-31732015000300008

Montell, G. (2003a). What's Your Philosophy on Teaching, and Does it Matter? The Chronicle of Higher Education. Disponible en: http://chronicle. com/article/Whats-Your-Philosophy-on-T/45132/

Montell, G. (2003b). How to Write a Statement of Teaching Philosophy. The Chronicle of Higher Education. Disponible en: http://chronicle.com/article/How-toWrite-a-Statement-of/45133/

Müller, M., Álamos, P., Meckes, L., Sanyal, A., y Cox, P. (2016). Percepción de estudiantes de pedagogía en relación a las oportunidades para el desarrollo de prácticas generativas en su formación. Estudios Pedagógicos, 42 (4), 145-163 https://scielo.conicyt. cl/pdf/estped/v42n4/art09.pdf

Pratt, D. (2005). Personal philosophies of teaching: A false promise? Academe, 91 (1), 32-35

Ramsey, J. y Fitzgibbons, D. (2005). Being in the classroom. Journal of Management Education, 29 (2), 333-356. DOI: /10.1177/1052562904271144

Russell, T. (2012). Paradigmatic changes in teacher education: The perils, pitfalls and unrealized promise of the reflective practitioner. Encounters on education, 13 , otoño 71-91

Russell, T. (2005a). Can reflective practice be taught? Reflective practice: International and 


\section{6 • ARTí́CuLOS CIENTíficos}

Multidisciplinary Perspectives, 6 (2), 199-204. DOI: /10.1080/14623940500105833

Russell, T. (2005b). The place of the practicum in preservice teacher education programs: Strengths and weaknesses in alternative assumptions about the experiences of learning to teach. En Garry Hoban (ed.), The missing links in teacher education design, 135-152. Holanda: Springer

Russell, T. y Munby, H. (1991). Reframing. The role of experience in developing teachers "professional knowledge". En Donald Schön (ed.) The reflective turn: case studies in and on educational practice, 164-187. New York: Teaches College Press

Schönwetter, D.; Sokal, L.; Friesen, M. y Taylor, K. L. (2002). Teaching philosophies reconsidered: A conceptual model for the development and evaluation of teaching philosophy statements. International Journal for Academic Development, 7 (1), 83-97. DOI:/10.1080/13601440210156501

Schön, D. (1983). The reflective practitioner: How professionals think in action. New York: Basic Books

Seldin, P. (1997). The Teaching Portfolio. Bolton: Anker Publishing Company

Staal, I., Klette, K., y Hamerness, K. (2018). Grounding Teacher Education in Practice Around the World: An Examination of Teacher Education Coursework in Teacher Education Programs in Finland, Norway, and the United States. Journal of Teacher Education 69 (2):184-197. DOI: 10.1177/0022487117728248
Thomas, L. (2012). What is Canadian about Teacher Education in Canada?, Canadian Association for Teacher Education. Disponible en: https://www. researchgate.net/publication/292996184_What_is Canadian_about_Teacher_Education_in_Canada_ Multiple_Perspectives_on_Canadian_Teacher_ Education_in_the_Twenty-First_Century

Ward, J. y McCotterb, S. (2004). Reflection as a visible outcome for preservice teachers. Teaching and Teacher Education, 20 (3), 243-257. Disponible en: http://ac.els-cdn.com/S0742051X04000137/1-s2.0S0742051X04000137-main.pdf?_tid=38074708189d-11e7-99cf-00000aacb362yacdnat $=14912450$ 98_10b01107d38f4b7ed4f627cb51708d77

Zeichner, K. (2012). The turn once again toward practice-based teacher education. Journal of Teacher Education, 63 (5), 376-382. DOI:/10.1177/0022487112445789

Zeichner, K. (2010). Rethinking the connections between campus courses and field experiences in College- and University-based teacher education. Journal of Teacher Education, 61 (89), 89-99. DOI:/10.1177/0022487109347671

Zeichner, K. (1996). Teachers as reflective practitioners and the democratization of schools reform. En Kenneth Zeichner, Susan Lee Melnick y Mary Louise Gomez (eds.), Currents of reform in preservice teacher education, 199-214. New York: Teachers College Press 